\title{
Altered energy dissipation ratio of the plantar soft tissues under the metatarsal heads in patients with type 2 diabetes mellitus: A pilot study
}

\author{
Chih-Chin Hsu ${ }^{\text {a }}$, Wen-Chung Tsai ${ }^{\text {a }}$, Yio-Wha Shau ${ }^{\text {b,* }}$, Kay-Lun Lee ${ }^{c}$, Ching-Fang Hu ${ }^{\text {a }}$ \\ ${ }^{a}$ Department of Physical Medicine and Rehabilitation, Chang Gung Memorial Hospital, School of Medicine, Chang Gung University, Taoyuan, Taiwan \\ ${ }^{\mathrm{b}}$ Institute of Applied Mechanics, Industrial Technology Research Institute, Angiogenesis Research Center, \\ National Taiwan University, No. 1, Roosevelt Road, Sec. 4, Taipei 106, Taiwan \\ ${ }^{\mathrm{c}}$ Department of Endocrinology and Metabolism, Chang Gung Memorial Hospital, Taoyuan, Taiwan
}

Received 20 October 2005; accepted 21 June 2006

\begin{abstract}
Background. Foot ulceration occurs frequently on the plantar aspect of the metatarsal head region, in which the altered foot biomechanics has been mentioned as a contributor. This study attempted to compare the energy dissipation in the plantar soft tissue under the metatarsal head between type 2 diabetic patients and age-matched healthy subjects in vivo.

Methods. The plantar soft tissues under the metatarsal heads in each left foot of 13 patients with type 2 diabetes mellitus and eight age-matched healthy subjects were measured with a loading-unloading device. The system comprised a 5-12 MHz linear-array ultrasound transducer and a load cell that operated at an impact velocity of about $5 \mathrm{~cm} / \mathrm{s}$. The stress-strain plot was derived by simultaneously recording the stress response and tissue deformation during a loading-unloading cycle. The energy dissipation ratio in all subjects could then be analyzed.

Findings. Although only the plantar soft tissue under the fourth metatarsal head in the diabetic patients endured significantly greater energy $(P=0.035)$ than the healthy subjects, a trend of an increased energy dissipation ratio for the metatarsals in the diabetic patients was observed.

Interpretation. The plantar soft tissue under the metatarsal head in the diabetic patients endures high dissipated energy during a simulating walking status in the study. The increased dissipated energy in the tissue may be responsible for the tissue breakdown in the diabetic patients.
\end{abstract}

(c) 2006 Elsevier Ltd. All rights reserved.

Keywords: Diabetic foot; Biomechanics; Metatarsus; Ultrasonography

\section{Introduction}

Foot ulceration resulting in amputation is one of the most devastating consequences of diabetes mellitus (Boulton, 1988). The tissue breakdown occurs frequently on the plantar aspect of the metatarsal head region (Reiber et al., 1998) and is caused by multiple factors, in which the altered foot biomechanics has been mentioned as a

\footnotetext{
* Corresponding author.

E-mail addresses: shau0014@itri.org.tw, ywshau@spring.iam.ntu.edu. tw (Y.-W. Shau).
}

contributor (Cevera et al., 1997). The importance of identifying tissue biomechanics lies in its ability to indicate the presence of a disease, as well as the basic information about the material itself (Garra et al., 1997). Therefore quantifying the plantar soft tissue properties under the metatarsal heads with a non-invasive assessment technique is important in screening patients at risk for developing an ulcer.

The plantar soft tissue thickness under the metatarsal head has been evaluated with ultrasonography (Cavanagh, 1999; Gooding et al., 1986; Zheng et al., 2000), computed tomography (Robertson et al., 2002) and roentgenography 
(Dreeben et al., 1987), and the results in diabetic patients varied from $0.6 \mathrm{~cm}$ to $1.4 \mathrm{~cm}$ (Gooding et al., 1986; Robertson et al., 2002; Zheng et al., 2000). Young's modulus of the plantar soft tissue between two adjacent metatarsal heads has been measured with the magnetic resonance imaging combined with an indentation design (Gefen et al., 2001). The ultrasound indentation system (Zheng et al., 2000), the 3-D indentor system (Klaesner et al., 2002) and the durometer (Brink, 1995) have been developed to assess the metatarsal tissue stiffness in the diabetic patients. Increased plantar soft tissue stiffness in diabetic patients has been found. However, the wide range of tissue stiffness may be due to different loading velocities and different maximum stress. Also, insufficient clinical information for the diabetic patients had been provided in those previous studies.

Energy dissipation ratio (EDR) has been proposed to be a parameter that capability of demonstrating the non-linear tissue characteristics (Hsu et al., 2000) and is less affected as the maximum stress exceeds $50 \mathrm{kPa}$ (Hsu et al., 1998; Wang et al., 1999a,b). Although those previous investigations have provided valuable references about the biomechanical behavior of plantar soft tissues, little is known about the EDR of the soft tissues under the metatarsal heads of diabetic patients. Further investigation is warranted to improve the knowledge of foot biomechanics for diabetic patients and the findings may assist in both prevention and treatment of the diabetic foot problem.

The real-time high-resolution ultrasonography can directly visualize and continuously monitor the deformation of the plantar soft tissue during a loading-unloading cycle. The purpose of the present work was to compare the EDR of plantar soft tissues under metatarsal heads between patients with type 2 diabetes mellitus and age-matched healthy subjects in vivo by combining ultrasonography and a load cell.

\section{Methods}

Thirteen patients (nine men, four women) with type 2 diabetes mellitus, fulfilling the diagnostic criteria of the American Diabetes Association (American Diabetes Association, 1997), and eight age-matched healthy subjects (five men, three women), the control group, were enrolled in this study. The subsequent data were presented all in the format of mean and standard error of mean (SEM). The mean age and body mass index (BMI) were 52.1 (SEM, 2.8) years old and 24.3 (SEM, 1.1) kg/m² respectively in the control subjects. The mean age and BMI were 53.4 (SEM, 2.6) years old and 25.4 (SEM, 1.3) kg/m² respectively in the diabetic patients. These volunteers did not have any foot problem within recent 6 months. All subjects gave informed consent before this non-invasive examination and the ethics committee approved this study.

The mean disease duration was 6.6 (SEM, 2.5) years and the average level of glycohemoglobin was 9.7 (SEM, 0.6)\% (range: $7.5-11.1 \%$ ) in the diabetic patients. Subjects unable to sense a 10-g Semmes-Weinstein monofilament (North Coast Medical Inc., San Jose, CA, USA) over the middle area of each metatarsus was defined to have significant diabetic neuropathy (Laing, 1994). None of the diabetic patients had callus problems, significant diabetic neuropathy and decreased pulsation of the dorsalis pedis and the posterior tibial arteries. Three diabetic patients had

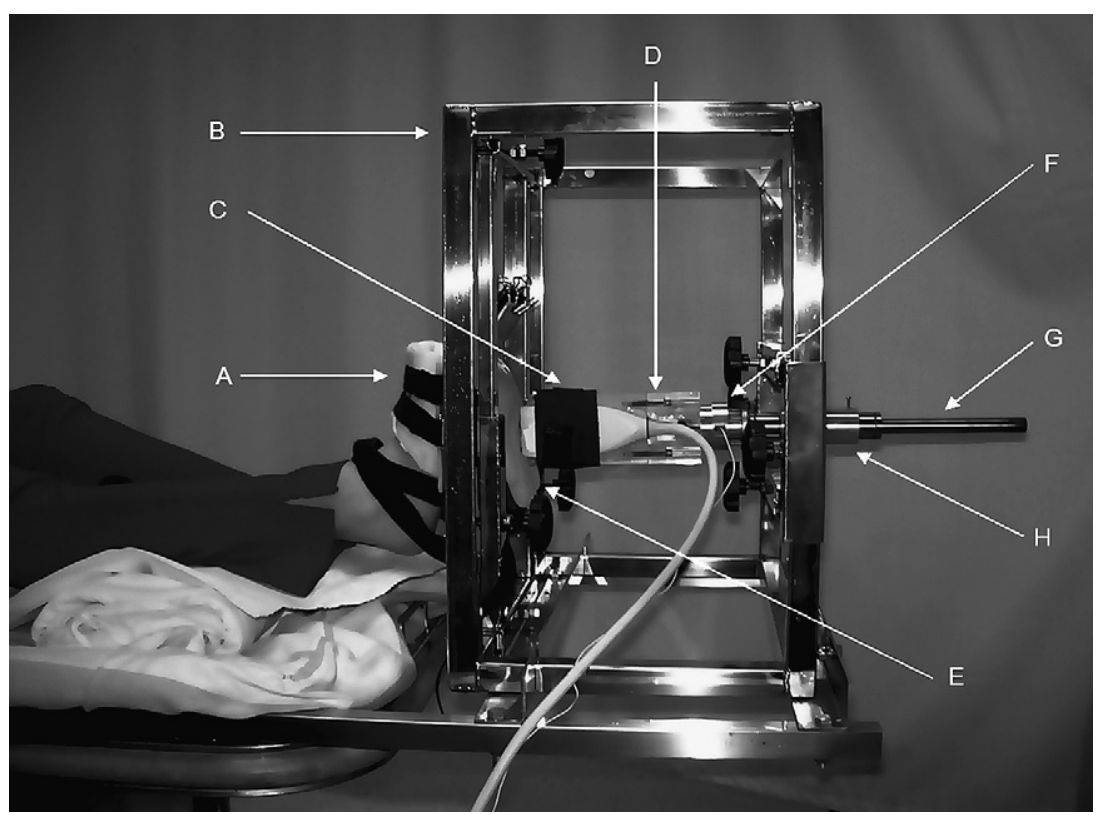

Fig. 1. Experimental design of this study. The tested foot was placed in a plastic cover (A), which was mounted on a stainless steel frame (B). Ultrasound transducer $(\mathrm{C})$ contacted with the examined region through a water bag (E). The transducer was further connected with a load cell (F) via an adapter (D). A hand-held steel rod $(\mathrm{G})$ could move the transducer freely in a bearing $(\mathrm{H})$ at different maximum loading-unloading velocities. M-mode morphologies and piezoelectric signals were transmitted to the high-resolution ultrasound and an IBM-compatible personal computer respectively. 
background diabetic retinopathy and one of them suffered from trace proteinuria simultaneously.

A $\quad 5-12 \mathrm{MHz}$ linear-array ultrasound transducer (HDI5000, Advance Technology Laboratory, Bothell, WA, USA) with the contact area of $4.73 \mathrm{~cm}^{2}$ was incorporated into a steel rod containing a miniature load cell (LM10KA, Kyowa Electronic Instruments Corp., Kyowa, Japan). They were then mounted on a loading-unloading device, comprising a plastic fixation cover, stainless steel frame, and a bearing. The experimental design was demonstrated in Fig. 1. The hand-held steel rod moved the transducer to and fro to load and unload the metatarsus. The freehand examination had the benefit of immediate response when the subject complained discomfort during the testing procedure. Each subject was placed in the supine position with the left ankle in neutral and the left knee in straight positions. The skin of the tested metatarsus was pretreated with alcohol to facilitate ultrasound penetration into plantar soft tissues. The metatarsus was monitored continuously during loading-unloading tests by motion-mode (M-mode) at the sampling rate of $100 \mathrm{~Hz}$ (Fig. 2).
When the ultrasound transducer did not contact the skin, unloaded metatarsal soft tissue thickness (UMT) was measured from the skin surface to the nearest metatarsal head cortex on the sonogram. Afterward, the probe loaded the tested metatarsus gradually. A beep sound was generated as soon as the compression force reached $98 \mathrm{~N}$, and the applied force was relieved immediately with ultrasound transducer leaving the metatarsal skin. With a contact area of $4.73 \mathrm{~cm}^{2}$, the maximum stress was equivalent to $207 \mathrm{kPa}$. The EDR of the tested metatarsals was calculated based on the stress range from 0 to $207 \mathrm{kPa}$. The ultrasound transducer was put along each toe ray and contacted the ball of the foot via a water bag. The water bag was used to maintain the contact between the transducer and the tested skin, especially in the unloading process during loading-unloading cycles. In the unloading phase, as soon as the ultrasound transducer separated from the tested metatarsus, clear visual evidence could be observed with the aid of water bag. The tested metatarsus was about $5 \mathrm{~cm}$ below the water level and the water level in the bag was raised slightly (typically less than $0.5 \mathrm{~cm}$ ) during the loading process. The pressure acting on the tested

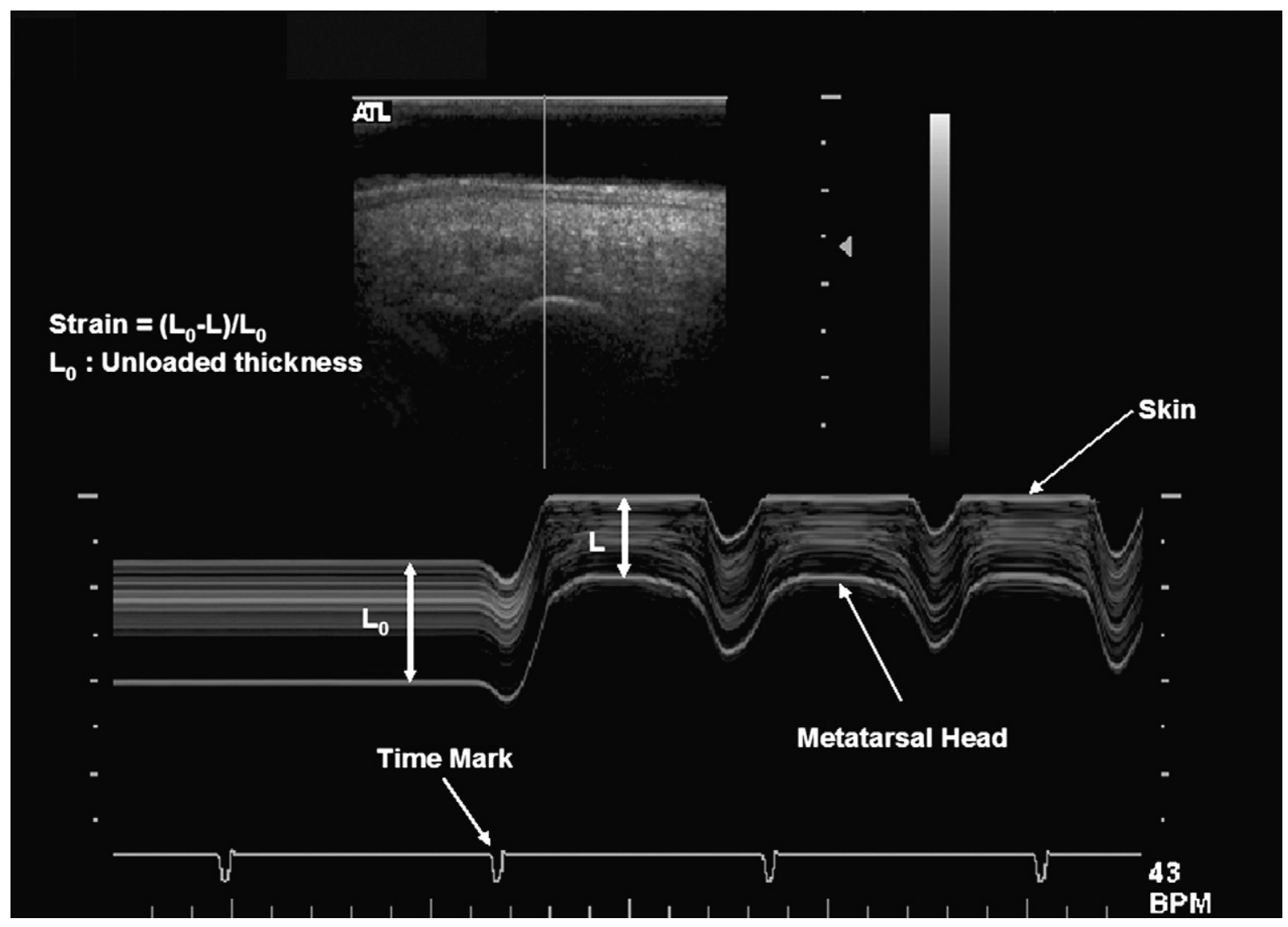

Fig. 2. B and M mode (upper panel) images of the plantar soft tissue under left third metatarsus in a 57-years-old healthy male. The vertical line in the B mode represented the ultrasound scanning line. The two sinusoid lines represented the skin and cortex of the third metatarsal head at different time in loading-unloading cycles. Unloaded plantar soft tissue thickness $\left(L_{0}\right)$ under the metatarsal head was measured from the skin to the nearest cortex. The thickness $(L)$ during a loading-unloading cycle could be measured from the M mode. Time marks produced by a pulse generator was used to synchronize with pressure waveforms. 
metatarsus by the water bag was about $500-550 \mathrm{~Pa}$, which was about $0.25 \%$ of the maximum loading pressure applied by the ultrasound transducer. Therefore, it was considered that the pressure on the tested metatarsus applied by the water bag could be neglected during the examination. All of the subjects were examined by an experienced operator.

The loading-unloading process was applied rhythmically according to the frequency of a metronome (Quartz Metronome SQ-77, Seiko S-Yard Co. Ltd., Tokyo, Japan) at $1 \mathrm{~Hz}$. Precondition of ten loading-unloading trials was done on each tested metatarsus before each examination to get a steady state of the soft tissue (Fung, 1993). Then, we examined the metatarsus with the mentioned loadingunloading period. Each metatarsus had a 10-min free of loading interval between each measurement. Impact velocity was defined as the velocity measured on the instant that the transducer contacted the skin. After the transducer contacting the metatarsus, the plantar soft tissue started to deform. The average loading rate was defined as the maximum strain of the plantar soft tissue divided by the loading time interval (Wang et al., 1999a,b). The mean impact velocity for the age matched healthy subjects and the diabetic patients was $5.26(\mathrm{SEM}, 0.13) \mathrm{cm} / \mathrm{s}$ (range: $3.27-6.71 \mathrm{~cm} / \mathrm{s}$ ) and 5.24 (SEM, 0.09) $\mathrm{cm} / \mathrm{s}$ (range: $4.02-$ $6.64 \mathrm{~cm} / \mathrm{s}$ ), respectively. The average loading rate for the age matched healthy subjects and the diabetic patients was 1.75 (SEM, 0.06) cm/s (range: $1.21-2.58 \mathrm{~cm} / \mathrm{s}$ ) and 1.82 (SEM, 0.04) cm/s (range: $1.26-2.66 \mathrm{~cm} / \mathrm{s}$ ) respectively.

Load cells analog signals were magnified by an amplifier (INA128/UAF42, Texas Instruments, Austin, TX, USA), in which frequency above $10 \mathrm{~Hz}$ was filtered, thus the electronic noise and irrelevant motion artifacts could be removed. The force signals were digitalized by an analogue-to-digital converter card (PCI-9118 DG, AD Link Technology Inc., Taipei, Taiwan) firstly and were then transmitted to an IBM compatible personal computer. A pulse generator was used to synchronize the force and tissue thickness information. The force information was further transformed into the pressure signals by dividing the contact area. Pressure waveforms and tissue deformation during a loading-unloading cycle were further analyzed and a stress-strain plot could be derived.

In a stress-strain relationship, the tested metatarsus showed non-linear stiffness in a stress-strain curve (Fig. 3). The upper loading curve and the lower unloading curve formed a closed area representing the dissipated energy. The energy dissipation ratio (EDR) of plantar soft tissues under metatarsal heads was given as

$$
\begin{aligned}
\mathrm{EDR}= & (\text { Bounded area/area under the loading curve }) \\
& \times 100 \%(\text { Bennett and Ker, 1990) }
\end{aligned}
$$

The area was estimated by numerical integration based trapezoid rule using the Microsoft Excel (Microsoft Corp., Redmond, WA, USA).

Reliability was determined by a test-retest procedure. We repeated the measurement on the plantar soft tissue

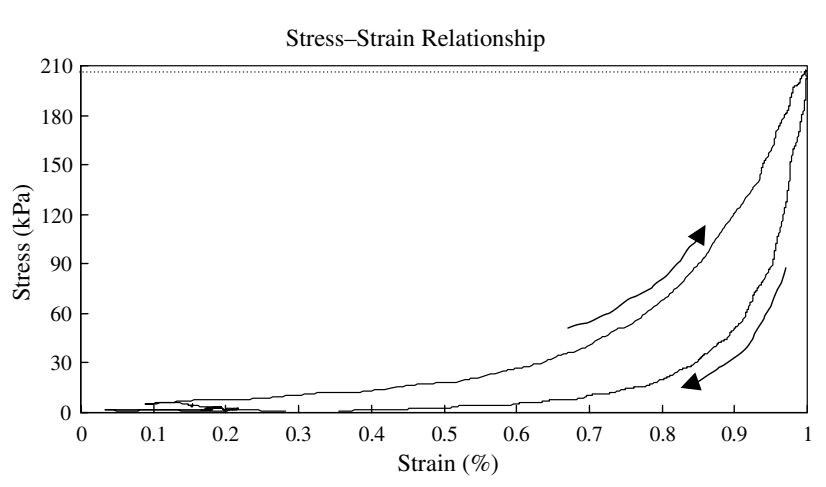

Fig. 3. The stress-strain curve of the plantar soft tissue under the left third metatarsus in a 62-years-old male diabetic patients. The $X$-axis and $Y$-axis represented the normalized strain [(unloaded thickness - thickness under certain stress)/(unloaded thickness - thickness under the maximum stress)] and stress respectively. The upper loading curve $(\nearrow)$ and the lower unloading curve $(\swarrow)$ form a closed area. The energy dissipation ratio is defined as the ratio of this closed area to the area formed by the loading curve, the vertical line, and the $X$-axis. The dotted line represented the maximum loading stress of $207 \mathrm{kPa}$.

under the second metatarsal head of the left foot in five healthy individuals at intervals of $10 \mathrm{~min}, 2 \mathrm{~h}$, and 1 week after the first measurement. Mann-Whitney U test, a nonparametric analysis, was used to compare the age, BMI, UMT, EDR, impact velocities and strain rates between the two groups. The Fisher's exact test was used to evaluate gender distribution between the two groups. The relationship between the age, BMI, UMT and EDR was analyzed by the Pearson correlation. A $P$-value of less than 0.05 is considered as statistically significant.

\section{Results}

The age, BMI and gender distribution were similar between the two groups $(P>0.05)$. The coefficient of variance $(\mathrm{CoV})$ for the UMT was then calculated from the 20 measurements and ranged from $1 \%$ to $3 \%$. The $\mathrm{CoV}$ for the energy dissipation ratio calculated from the 20 measurements ranged from $2.56 \%$ to $8.45 \%$. The mean UMT had a decrease trend from the first to the fifth metatarsus, with the values of 1.41 (SEM, 0.05), 1.35 (SEM, 0.07), 1.26 (SEM, 0.04), 1.16 (SEM, 0.03), 1.12 (SEM, 0.07) cm in the control group and 1.37 (SEM, 0.06), 1.34 (SEM, 0.09), 1.28 (SEM, 0.09), 1.27 (SEM, 0.10), 1.27 (SEM, $0.06) \mathrm{cm}$ in the diabetic patients group. There was no significant difference of the UMT between the two groups $(P>0.05)$. The impact velocities and loading rates were not significantly different $(P>0.05)$ between the two groups, either.

The plantar soft tissue under the fourth metatarsal head in the diabetic patients had significantly greater EDR than that in the healthy controls. However, a trend of increased EDR for the metatarsals in the diabetic patients was observed. The measurement for all the metatarsals in the two groups was presented in Table 1.

No correlation could be found between the age, BMI, UMT and EDR of all the metatarsals, except a fair 
Table 1

Energy dissipation ratio of the plantar soft tissues under the metatarsal heads in the two groups

\begin{tabular}{llll}
\hline & \multicolumn{3}{l}{ EDR $(\%)$} \\
\cline { 2 - 4 } & Control & DM & $P$-value \\
\hline MT1 & $61.7(2.8)$ & $62.2(3.7)$ & 0.778 \\
MT2 & $58.7(2.4)$ & $62.0(2.4)$ & 0.438 \\
MT3 & $59.0(2.2)$ & $61.8(1.9)$ & 0.351 \\
MT4 & $54.5(2.8)$ & $62.2(1.8)$ & 0.035 \\
MT5 & $56.0(4.1)$ & $63.0(2.3)$ & 0.270 \\
\hline
\end{tabular}

The standard error of mean for the energy dissipation ratio (EDR) values is given in parentheses.

Control: age-matched healthy subjects.

DM: patients with type 2 diabetes mellitus.

MT1-5: the plantar soft tissue under the first to the fifth metatarsus.

${ }^{a}$ Mann-Whitney $U$ test for estimation of differences between groups.

correlation $(P=0.02, r=0.576)$ between the EDR of the fourth metatarsus and the BMI.

\section{Discussion}

The reported normal walking gait period ranged from 0.98 to $1.2 \mathrm{~s}$ (DuChatinier et al., 1970; Murray et al., 1969). In-shoe peak plantar pressure at metatarsal heads, evaluated at walking speed of $1.66 \mathrm{~m} / \mathrm{s}$ with capacitancebased pressure measuring insoles, ranged from $185 \mathrm{kPa}$ to $198 \mathrm{kPa}$ (Rozema et al., 1996). In this study, the loadingunloading period was one second and the maximum compression stress on each tested metatarsus was $207 \mathrm{kPa}$. Therefore, plantar soft tissue properties in the study were measured at the condition close to the physiological walking status.

The plantar soft tissue thickness under the metatarsal head had been evaluated in one healthy person and one patient with metatarsalgia by radiography (Dreeben et al., 1987). Magnification caused by divergence of the $\mathrm{X}$-ray beam may distort the true plantar soft tissue thickness under the metatarsal head. The soft tissue thickness under the metatarsal head had been measured with transversely oriented ultrasound (Gooding et al., 1986; Graf et al., 1988). Gooding et al., (1986) placed the 10-MHz ultrasound transducer transversely on the forefoot to survey several metatarsals simultaneously in 24 normal controls, 38 diabetic patients without foot ulcers and 11 diabetic patients with foot ulcers. Graf et al. (1988) used ultrasound to determine the metatarsal head position in eight patients with suspected plantarflexed metatarsals and associated painful pedal callosities. Their results showed that the metatarsal thickness reached its maximum beneath the second metatarsal head and its minimum beneath the first and fifth rays. In their studies, the thickness under the first metatarsal head was measured from the skin surface to the sesamoid bone, resulting in a thinner tissue thickness. Furthermore, this technique may have missed actual metatarsal heads because they aligned in a curve on the horizontal plane.
The plantar soft tissue thickness of healthy subjects (aged 30) had been measured with longitudinally oriented ultrasound transducer and decreased progressively from the first to the fifth metatarsus at non-weight bearing condition (Wang et al., 1999a,b). Similar examination had been done for the plantar soft tissue thickness in nine healthy young adults and 10 healthy elder persons, and no difference between them had been reported (Hsu et al., 2005). The mean UMT had a decrease trend from the first to the fifth metatarsus in the study, in which the tendency was similar to the previous works (Dreeben et al., 1987; Hsu et al., 2005; Wang et al., 1999a,b). No difference in the plantar soft tissue thickness between the diabetic patients (mean age of 55) and the age-matched healthy persons was found after examining 32 healthy persons and 16 diabetic patients with the computed tomography (Robertson et al., 2002). The study had similar findings probably due to the close glycohemoglobin level between the two investigations. However different observations for the plantar soft tissue changes in diabetic patients do exist. Zheng et al. (2000) used A-mode ultrasound to examine plantar soft tissue in four healthy persons aged about 22-yearsold and four diabetic patients aged about 63-years-old. The diabetic patients were found to have thinner forefoot fat pad and the result was similar to the previous report (Gooding et al., 1986). Apparently, different measurement methods and patient status may lead to different findings.

The distance between the skin and the metatarsal head was continuously monitored during the loading-unloading process in the study. The minor movement of the tested metatarsal head has been constrained by a plastic cover in order to make an accurate measurement of the metatarsal soft tissue thickness during the loading-unloading process. In the stress-strain relationship of a viscoelastic material, the loading and unloading curves typically run in different paths and show a non-linear characteristic. These two curves form an enclosed area representing the dissipated energy of the testing material. The EDR is calculated from the difference of the area under the loading and unloading curves and is considered to be an adequate indicator in reflecting the non-linear tissue characteristic (Hsu et al., 2002).

Although limited number of subjects was included in the study, there seemed to be a trend that the plantar soft tissues under all the five metatarsal heads in diabetic patients have absorbed greater energy than those in the age-matched healthy subjects in a loading-unloading test. The observation is similar as findings concerning the heel-pad EDR between diabetic patients and age-matched healthy subjects (Hsu et al., 2000). Poor rebound of the unloading process, i.e., prolonging time to recover the shapes in the plantar soft tissue (Hsu et al., 2002), may contribute to the increased EDR in the metatarsals of the diabetic patients. The plantar soft tissue under the metatarsal head had higher EDR than that of the healthy young persons, and this phenomenon may eventually lead to the development of metatarsalgia in the aging people 
(Hsu et al., 2005). An increase of EDR in the injured heel pads reconstructed with musculocutaneous flaps had been proposed to be the culprit for the development of shockinduced heel discomfort and ulceration (Wang et al., 1999a,b). Perhaps an increased EDR damages the metatarsal and increases the susceptibility to foot ulceration in diabetic patients.

The UMT and EDR in most of the metatarsals did not correlate with the BMI in the study. The findings were similar to the results in the measurement of the heel-pad mechanical properties in diabetic patients and age-matched healthy subjects (Hsu et al., 2000) and the analysis for effects of aging on the metatarsus (Hsu et al., 2005). In the study for plantar soft tissues properties in young persons, EDR increased significantly with weight (Wang et al., 1999a,b). Further investigation between the plantar soft tissue behavior and the BMI may be necessary to reach a conclusion for their relationship. No correlation with the UMT could be expected, because the EDR has been normalized with its initial thickness.

Systemic tissue changes are already known to accompany long-term diabetes in all patients with diabetes mellitus. Since the plantar soft tissue is a continuous structure throughout the forefoot and heel region, it is believed that the same or similar tissue changes that occur in the heel pad also occur in the plantar soft tissues under the metatarsal heads. Distorted and ruptured collagen fibers and atrophic fat globules have been observed in diabetic heel pads (Buschmann et al., 1995). The collagen fibrils in diabetic heel pads were involved in varied areas at varied extents. The typical band periodicity of each involved collagen fibrils disappeared (Hsu et al., 2002). The deleterious effects of glycation, a non-enzymatic reaction, on collagen play central roles in the evolution of disorganized collagen fibrils (Paul and Bailey, 1996). These qualitative changes of collagen fibrils may influence tissue properties of the plantar soft tissues under the metatarsal heads (Hsu et al., 2000).

The small sample size may limit the study power and only a nonparametric analysis was allowed to estimate the tested results. Nonparametric analysis assays data without assuming an underlying distribution, which can avoid the potentially large errors brought about by making incorrect assumptions. However, the confidence bounds associated with the nonparametric analysis are usually much wider than those calculated via parametric analysis and the predictions of study power are not possible. Although a trend of increased energy dissipation in plantar soft tissues under all five metatarsal heads in the diabetic patients was observed, only the fourth metatarsus in the diabetic patients had significantly higher EDR than that in the age-matched healthy subjects. Perhaps these patients did not have diabetes long enough to develop differences in soft tissue function. Although the tissue stiffness determined from the unloaded and end-loaded conditions cannot present the non-linear stress-strain characteristics of the metatarsal soft tissue, curve fit methods, including bilinear fit or non-linear fit, may scientifically document the non-linear tissue properties. This work is interesting and may be helpful in describing the soft tissue mechanical properties in the future study.

\section{Conclusion}

This study shows that the plantar soft tissues under the metatarsal heads in the diabetic patients endure high dissipated energy, which may lead to the further tissue breakdown. Therefore, adequate footware may be indicated in diabetic patients to prevent the development of the foot ulceration.

\section{Acknowledgements}

This study was granted by our Department of Industrial Technology, Ministry of Economic Affairs for pure academic research. The authors thank Dr. Kuang-Hung Hsu, a statistician of the Health Care Management, Chang Gung University, for providing suggestion on the statistic analysis.

\section{References}

American Diabetes Association, 1997. Report of the Expert Committee on the diagnosis and classification of diabetes mellitus. Diabetes Care 20, pp. 1183-1197.

Bennett, M.B., Ker, R.F., 1990. The mechanical properties of the human subcalcaneal fat pad in compression. J. Anat. 171, 131-138.

Boulton, A.J., 1988. The diabetic foot. Med. Clin. North Am. 72, 1513 1530.

Brink, T., 1995. Induration of the diabetic foot pad: another risk factor for recurrent neuropathic plantar ulcers. Biomed. Technol. 40, 205-209.

Buschmann, W.R., Jahss, M.H., Kummer, F., Desai, P., Gee, R.O., Ricci, J.L., 1995. Histology and histomorphometric analysis of the normal and atrophic heel fat pad. Foot Ankle Int. 16, 254-258.

Cavanagh, P.R., 1999. Plantar soft tissue thickness during ground contact in walking. J. Biomech. 32, 623-628.

Cevera, J.J., Bolton, L.L., Kerstein, M.D., 1997. Options for diabetic patients with chronic heel ulcers. J. Diabet. Complicat. 11, 358-366.

Dreeben, S., Thomas, P.B., Noble, P.C., Tullos, H.S., 1987. A new method for radiography of weight-bearing metatarsal heads. Clin. Orthop. Relat. Res. 224, 260-267.

Du Chatinier, K., Molen, N.H., Rozendal, R.H., 1970. Step length, step frequency and temporal factors of the stride in normal human walking. Proc. Kon. Ned. Akad. Wat. C 73, 214-227.

Fung, Y.C., 1993. Biomechanics: mechanical properties of living tissues, second ed. Springer-Verlag, New York.

Garra, B.S., Cespedes, E.I., Ophir, J., Spratt, S.R., Zuurbier, R.A., Magnant, C.M., Pennanen, M.F., 1997. Elastography of breast lesions: initial clinical results. Radiology 202, 79-86.

Gefen, A., Megido-Ravid, M., Azariah, M., Itzchak, Y., Arcan, M., 2001. Integration of plantar soft tissue stiffness measurements in routine MRI of the diabetic foot. Clin. Biomech. 16, 921-925.

Gooding, G.A., Stess, R.M., Graf, P.M., Moss, K.M., Louie, K.S., Grunfeld, C., 1986. Sonography of the sole of the foot: evidence for loss of foot pad thickness in diabetes and its relationship to ulceration of the foot. Invest. Radiol. 21, 45-48.

Graf, P.M., Farac, K., Stess, R.M., Gooding, G.A., 1988. Highresolution ultrasound in the preoperative and postoperative assessment of distal metatarsal osteotomy: preliminary report. Invest. Radiol. 23, 827-831. 
Hsu, T.C., Wang, C.L., Tsai, W.C., Kuo, J.K., Tang, F.T., 1998. Comparison of the mechanical properties of the heel pad between young and elderly adults. Arch. Phys. Med. Rehab. 79, 1101-1104.

Hsu, T.C., Wang, C.L., Shau, Y.W., Tang, F.T., Li, K.L., Chen, C.Y., 2000. Altered heel-pad mechanical properties in patients with type 2 diabetes mellitus. Diabet. Med. 17, 854-859.

Hsu, T.C., Lee, Y.S., Shau, Y.W., 2002. Biomechanics of the heel pad for type 2 diabetic patients. Clin. Biomech. 17, 291-296.

Hsu, C.C., Tsai, W.C., Chen, C.P.C., Shau, Y.W., Wang, C.L., Chen, M.J.L., Chang, K.J., 2005. Effects of aging on the plantar soft tissue properties under the metatarsal heads at different impact velocities. Ultrasound Med. Biol. 31, 1423-1429.

Klaesner, J.W., Hastings, M.K., Zou, D., Lewis, C., Mueller, M.J., 2002. Plantar tissue stiffness in patients with diabetes mellitus and peripheral neuropathy. Arch. Phys. Med. Rehab. 83, 1796-1801.

Laing, P., 1994. Diabetic foot ulcers. Am. J. Surg. 167, 31S-36S.

Murray, M.P., Kory, R.C., Clarkson, B.H., 1969. Walking patterns in healthy old men. J. Gerontol. 24, 169-178.

Paul, R.G., Bailey, A.J., 1996. Glycation of collagen: the basis of its central role in the late complications of aging and diabetes. Int. J. Biochem. Cell Biol. 28, 1297-1310.
Reiber, G.E., Lipsky, B.A., Gibbons, G.W., 1998. The burden of diabetic foot ulcers. Am. J. Surg. 176, 5S-10S.

Robertson, D.D., Mueller, M.J., Smith, K.E., Commean, P.K., Pilgram, T., Johnson, J.E., 2002. Structural changes in the forefoot of individuals with diabetes and a prior plantar ulcer. J. Bone Joint Surg. 84-A, 1395-1404.

Rozema, A., Ulbrecht, J.S., Pammer, S.E., Cavanagh, P.R., 1996. In-shoe plantar pressures during activities of daily living: implications for therapeutic footwear design. Foot Ankle Int. 17, 352-359.

Wang, C.L., Hsu, T.C., Shau, Y.W., Shieh, J.Y., Hsu, K.H., 1999a. Ultrasonographic measurement of the mechanical properties of the sole under the metatarsal heads. J. Orthop. Res. 17, 709-713.

Wang, C.L., Shau, Y.W., Hsu, T.C., Chen, H.C., Chien, S.H., 1999 b. Mechanical properties of heel pads reconstructed with flaps. J. Bone Joint Surg. 81-B, 207-211.

Zheng, Y.P., Choi, Y.K.C., Wong, K., Chan, S., Mak, A.F.T., 2000. Biomechanical assessment of plantar foot tissue in diabetic patients using an ultrasound indentation system. Ultrasound Med. Biol. 26, 451-456. 